\title{
Simulation of Analog Costas Loop Circuits
}

\author{
Roland E. Best ${ }^{1}$ Nikolay V. Kuznetsov ${ }^{2,3}$ Gennady A. Leonov ${ }^{3} \quad$ Marat V. Yuldashev $^{2,3}$ Renat V. Yuldashev ${ }^{2,3}$ \\ ${ }^{1}$ Best Engineering, Oberwil, Switzerland \\ ${ }^{2}$ Department of Mathematical Information Technology, University of Jyväskylä, Jyväskylä FI-40014, Finland \\ ${ }^{3}$ Department of Applied Cybernetics, Saint Petersburg State University, Saint Petersburg 198504, Russia
}

\begin{abstract}
The analysis of stability and numerical simulation of Costas loop circuits for the high-frequency signals is a challenging task. The problem lies in the fact that it is necessary to observe very fast time scale of input signals and slow time scale of signal's phases simultaneously. To overcome this difficulty, it is possible to follow the classical ideas of Gardner and Viterbi to construct a mathematical model of Costas loop, in which only slow time change of signal's phases and frequencies is considered. Such an construction, in turn, requires the computation of phase detector characteristic, depending on the waveforms of the considered signals. In this paper, the problems of nonlinear analysis of Costas loops and the approaches to the simulation of the classical Costas loop, the quadrature phase shift keying (QPSK) Costas loop, and the two-phase Costas loop are discussed. The analytical method for the computation of phase detector characteristics of Costas loops is described
\end{abstract}

Keywords: Phase-locked loop (PLL) based circuits, Costas loop, phase detector characteristic, simulation, nonlinear analysis.

\section{Introduction}

Nowadays, binary phase shift keying (BPSK) and quadrature phase shift keying (QPSK) modulation techniques are widely used in telecommunication ${ }^{[1,2]}$. For BPSK and QPSK data transmission, various modifications of the phase-locked loop (PLL) are used, e.g., circuits with a squaring device and the so called Costas loop ${ }^{[1,3-5]}$. Because the realization of squaring circuits can be quite difficult, the Costas loop is the preferred variant. In the following, we will concentrate on the Costas loop, which is easy for implementation and effective for demodulation. The Costas loop is a classical analog PLL based circuit for carrier recovery ${ }^{[6-8]}$. Nowadays, among the applications of Costas loop, there are global positioning systems $(\mathrm{GPS})^{[9,10]}$, wireless communication ${ }^{[11]}$ and others ${ }^{[8,12-19]}$.

Although the Costas loop is inherently a nonlinear control system, it is analyzed in most textbooks and papers by using linear models, which represent a simplification of reality (see a plenary lecture of D. Abramovich at American Control Conference 2002 $2^{[20]}$ ). The nonlinear analysis of PLL-based circuits is a difficult task (see [21-42]) hence numerical simulation is widely used in practice (see [1, 43-47] and others).

Complete numerical analysis of the physical model of PLL-based circuits is a very challenging task because it becomes necessary to observe simultaneously very fast signals (the high frequency signals) and slower signals (i.e., the demodulated data signals $)^{[48,49]}$. To analyze the high frequency signals accurately, a very high sampling rate is required, which makes it difficult to perform a simulation in a reasonable time.

Regular paper

Special Issue on Recent Advances on Complex Systems Control, Modelling and Prediction II

Manuscript received September 26, 2013; accepted October 11, 2013

This work was supported by Academy of Finland, Russian Ministry of Education and Science (Federal Target Program), and Russian Foundation for Basic Research and Saint-Petersburg State University.
In this paper, we will discuss simulation methods for various modifications of the Costas loop.

\subsection{Mathematical models of Costas loops}

In this section, various modifications of the Costas loop are discussed.

\subsubsection{Classical BPSK Costas loop}

The physical model of the classical BPSK Costas loop with the sinusoidal carrier and voltage-controlled oscillator (VCO) signals is shown in Fig. 1, where the input signal is the BPSK signal, which is the product of the slow data signal $m(t)= \pm 1$ and the harmonic carrier $\sin \left(\theta^{1}(t)\right)$ (the carrier period is several orders of magnitude smaller than the time between data transitions); $\theta^{1}(t)$ represents the carrier phase. In analogy, $\sin \left(\theta^{2}(t)\right)$ is the output signal of the VCO, and $\theta^{2}(t)$ represents its phase. The Hilbert transform block shifts the phase of the VCO output signal by $-90^{\circ}$. Block $\otimes$ is a multiplying block.

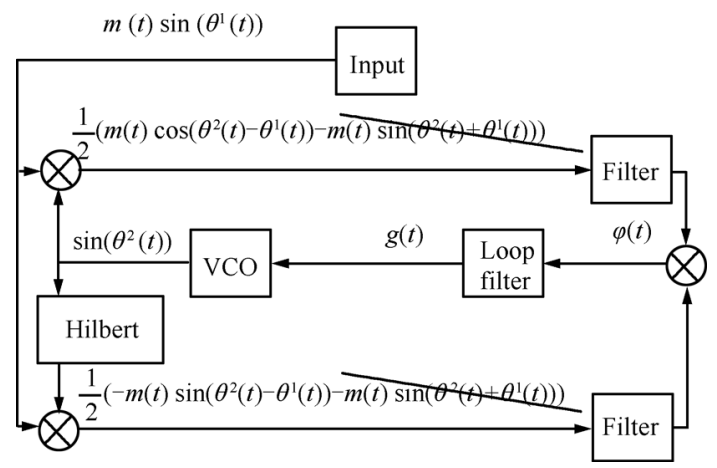

Fig. 1 Physical model of Costas loop in the signal/time space

The standard engineering assumption is that a low-pass filter removes the upper sideband whose frequency is about twice the carrier frequency and passes the lower sideband without change (ideal low-pass filter). Thus, the input of 
the loop filter can be approximated as

$$
\varphi(t) \approx \varphi(\theta(t))=\frac{1}{8} \sin (2 \theta(t))
$$

where $\theta(t)=\theta^{2}(t)-\theta^{1}(t)$. Function $\varphi(\theta)$ is called the phase detector (PD) characteristic of Costas loop for sinusoidal signals (phase detector is a nonlinear element, used to match the phases of reference and tunable signals). The loop filter output signal $g(t)$ adjusts the VCO frequency to the frequency of input signal carrier. If the system has acquired lock: $g(t)=0$ and $\theta^{1}(t)=\theta^{2}(t)$, then the output of the upper low-pass filter is the data signal $m(t)$.

\subsubsection{QPSK Costas loop}

Next, the Costas loop for QPSK ${ }^{[1]}$ is considered. To implement such a system, two carriers have to be generated in the transmitter that are offset in phase by $90^{\circ}$. The symbol stream $m(t)$ is partitioned into two data streams: One of them (e.g., $\left.m_{1}(t)\right)$ is used to modulate the in-phase carrier, while the other (e.g., $\left.m_{2}(t)\right)$ modulates the quadrature carrier. The combined output signal of the transmitter can be written as

$$
m_{1}(t) \cos \left(\theta^{1}(t)\right)-m_{2}(t) \sin \left(\theta^{1}(t)\right) .
$$

The receiver of the QPSK signal is shown in Fig. 2. In contrast to the classical Costas loop, the two low-pass filters in the upper and lower branches are now followed by limiters $(\operatorname{sgn}(\cdot))$. For the ideal low-pass filters, one gets

$$
\begin{aligned}
Q(t) & =\frac{1}{2}\left[m_{1}(t) \cos (\theta(t))-m_{2}(t) \sin (\theta(t))\right] \\
I(t) & =\frac{1}{2}\left[m_{1}(t) \sin (\theta(t))-m_{2}(t) \cos (\theta(t))\right] .
\end{aligned}
$$

If the system has acquired lock, then $Q(t)$ and $I(t)$ represent the data signal.

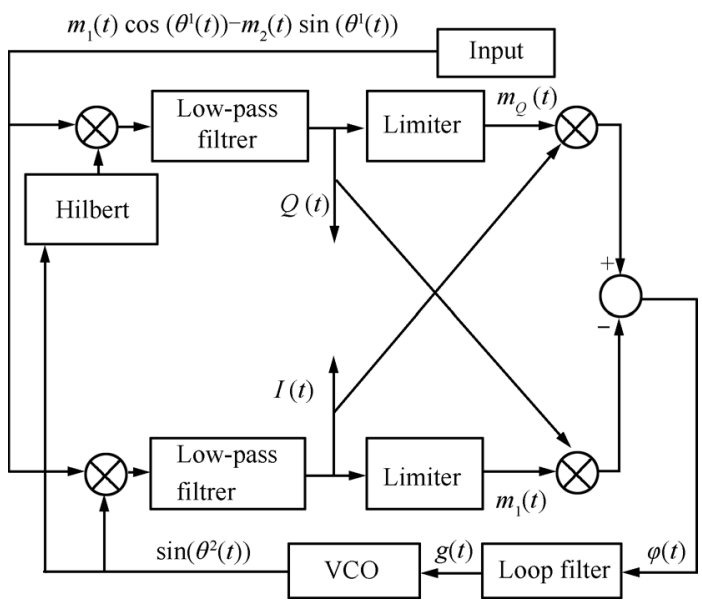

Fig. 2 QPSK Costas loop

If, for the sake of simplicity, one assumes that $m_{1}(t) \equiv$ $m_{2}(t) \equiv 1$, then the input of the loop filter can be approximated as

$$
\begin{aligned}
\varphi(t) \approx & \varphi(\theta(t))=0.5[\sin (\theta(t))+\cos (\theta(t))] \times \\
& \operatorname{sgn}[\cos (\theta(t))-\sin (\theta(t))]- \\
& 0.5[\cos (\theta(t))-\sin (\theta(t))] \operatorname{sgn}[\sin (\theta(t))+\cos (\theta(t))]=
\end{aligned}
$$

$$
\begin{cases}\sin (\theta(t)), & \text { if } \theta(t) \in\left[-\frac{\pi}{4}, \frac{\pi}{4}\right]+2 \pi k \\ -\cos (\theta(t)), & \text { if } \theta(t) \in\left[\frac{\pi}{4}, \frac{3 \pi}{4}\right]+2 \pi k \\ -\sin (\theta(t)), & \text { if } \theta(t) \in\left[\frac{3 \pi}{4}, \frac{5 \pi}{4}\right]+2 \pi k \\ \cos (\theta(t)), & \text { if } \theta(t) \in\left[-\frac{5 \pi}{4}, \frac{7 \pi}{4}\right]+2 \pi k .\end{cases}
$$

\subsubsection{Two-phase Costas loop}

Next, a modified implementation of the Costas loop ${ }^{[50]}$ is considered (see Fig. 3).

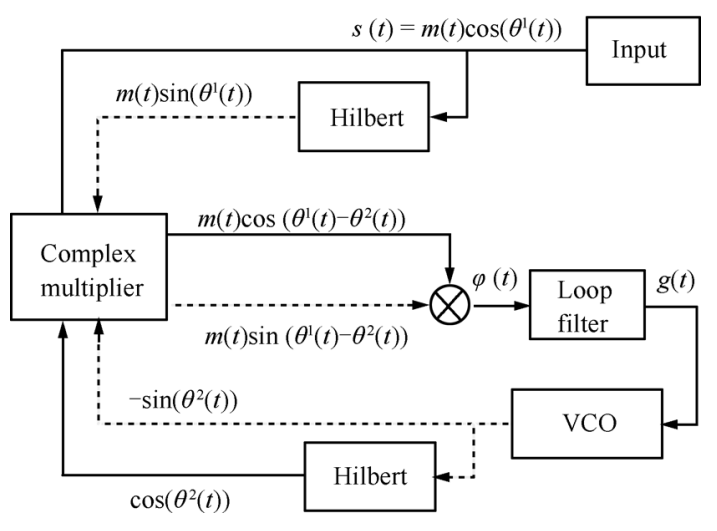

Fig. 3 Two-phase Costas loop

The input signal is again given by $s(t)=m(t) \cos \left(\theta^{1}(t)\right)$. This signal is converted into the so called pre-envelope signal $\hat{s}(t)$, which is given by $\hat{s}(t)=s(t)+\mathrm{j} H(s(t))$, where $H$ stands for the Hilbert transform, $\hat{s}(t)=\mathrm{e}^{\mathrm{j} \theta^{1}(t)}$ is a complex signal, $m(t)$ is the (slow) data signal and can have the values 1 or -1 . In contrast to the classical BPSK Costas loop, the VCO now also creates a complex output signal $\mathrm{e}^{-\mathrm{j} \theta^{2}(t)}$. Multiplying these two signals in the locked state yields the multiplier output signal $m(t)$. This has a very important consequence: There is no need for a low-pass filter at the output of the multiplier.

Fig. 4 represents the complex multiplier.

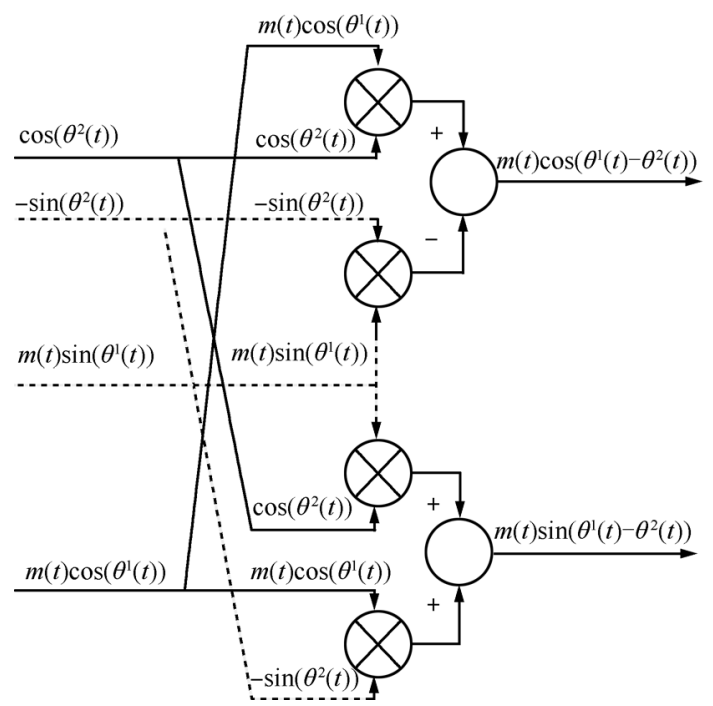

Fig. 4 Complex multiplier 
The outputs of the complex multiplier are the following

$$
\begin{aligned}
& m(t)\left[\cos \left(\theta^{1}(t)\right) \cos \left(\theta^{2}(t)\right)+\sin \left(\theta^{1}(t)\right) \sin \left(\theta^{2}(t)\right)\right]= \\
& m(t) \cos \left(\theta^{1}(t)-\theta^{2}(t)\right) \\
& m(t)\left[\sin \left(\theta^{1}(t)\right) \cos \left(\theta^{2}(t)\right)-\cos \left(\theta^{1}(t)\right) \sin \left(\theta^{2}(t)\right)\right]= \\
& m(t) \sin \left(\theta^{1}(t)-\theta^{2}(t)\right) .
\end{aligned}
$$

Therefore, the input of the loop filter takes the form

$$
\varphi(t)=\varphi(\theta(t))=\frac{1}{2} \sin (2(\theta(t))) .
$$

The two-phase Costas loop can also be extended for use in the QPSK data transmission.

\section{Simulation of Costas loops in Simulink}

Next, Simulink models of Costas loops are considered. Fig. 5 shows the model for the classical Costas loop for BPSK; Fig. 6 shows the model of the Costas loop for QPSK; and Fig. 7 represents the two-phase Costas loop.

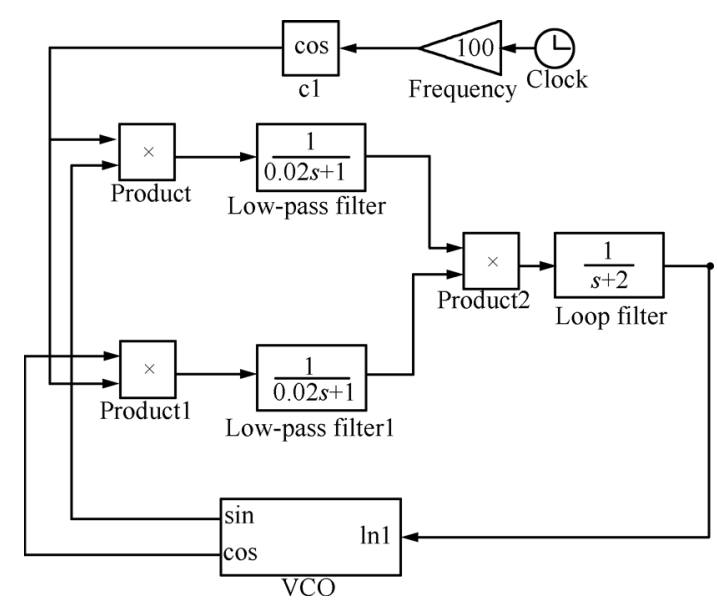

Fig. 5 Simulink model of the classical Costas loop

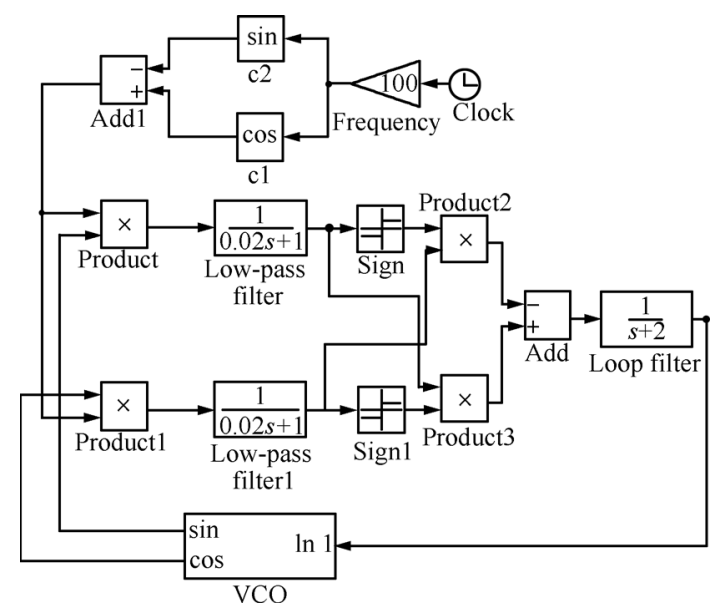

Fig. 6 Simulink model of the QPSK Costas loop

Despite the fact that filter transfer functions and other parameters are chosen the same, the transients are different. First, the frequency difference between the VCO freerunning frequency and the carrier frequency is chosen so low that all three circuits are able to get locked, as shown in Fig. 8.

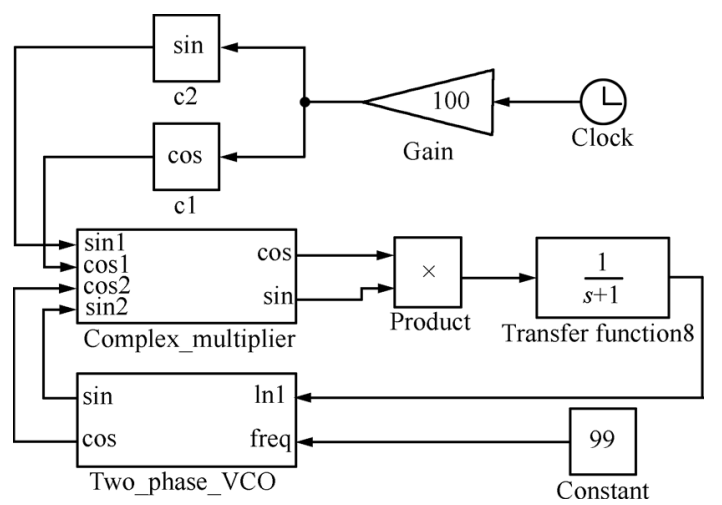

Fig. 7 Simulink model of the two-phase Costas loop
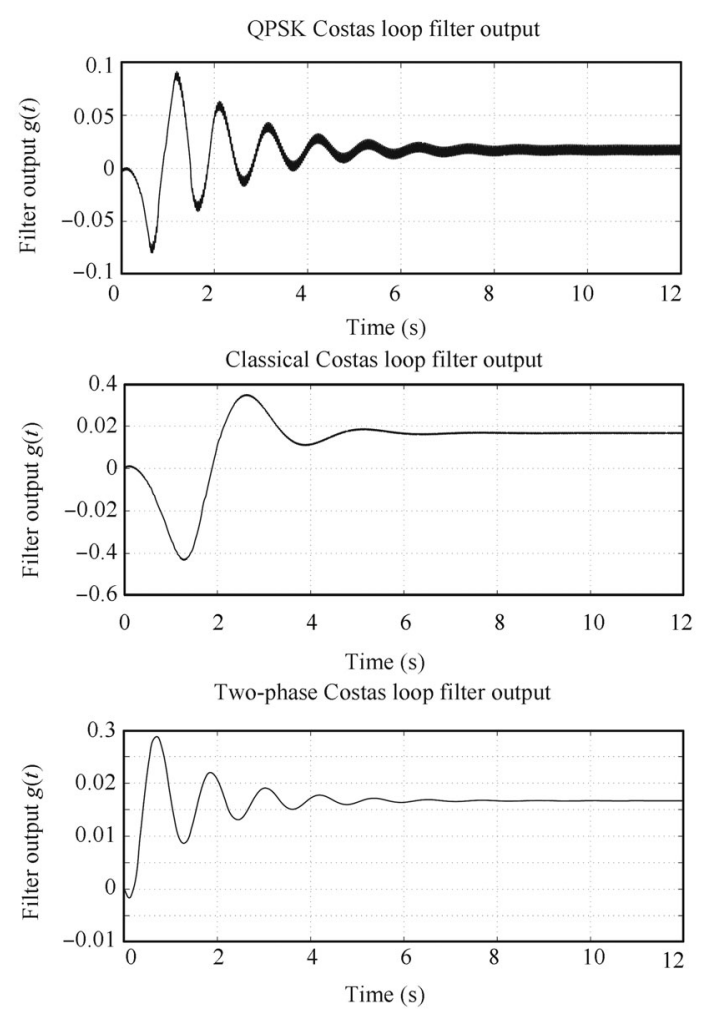

Fig. 8 Plot of the VCO input $g(t)$. VCO input gain: 30; lowpass filters transfer function: $\frac{1}{0.02 s+1}$; loop filter transfer function: $\frac{1}{s+2}$; VCO free-running frequency: 99.5 ; carrier frequency: 100

When the frequency difference between the VCO freerunning frequency and the carrier frequency is chosen such that it exceeds a value called pull-in range, the classical Costas loop for BPSK is no longer able to acquire lock. This is shown in Fig. 9. When the frequency difference is further increased, the QPSK Costas loop also goes out of lock (see Fig. 10). The same holds true for the two-phase Costas loop, which can be seen in Fig. $11^{1}$.

\footnotetext{
${ }^{1}$ The pull-in range of the modified Costas loop with lag-lead filter $\frac{1+\alpha s}{1+\beta s}$ will be larger, with the PI filter $\frac{1+\alpha s}{\beta s}$, the pull-in range may conceivably be infinite.
} 

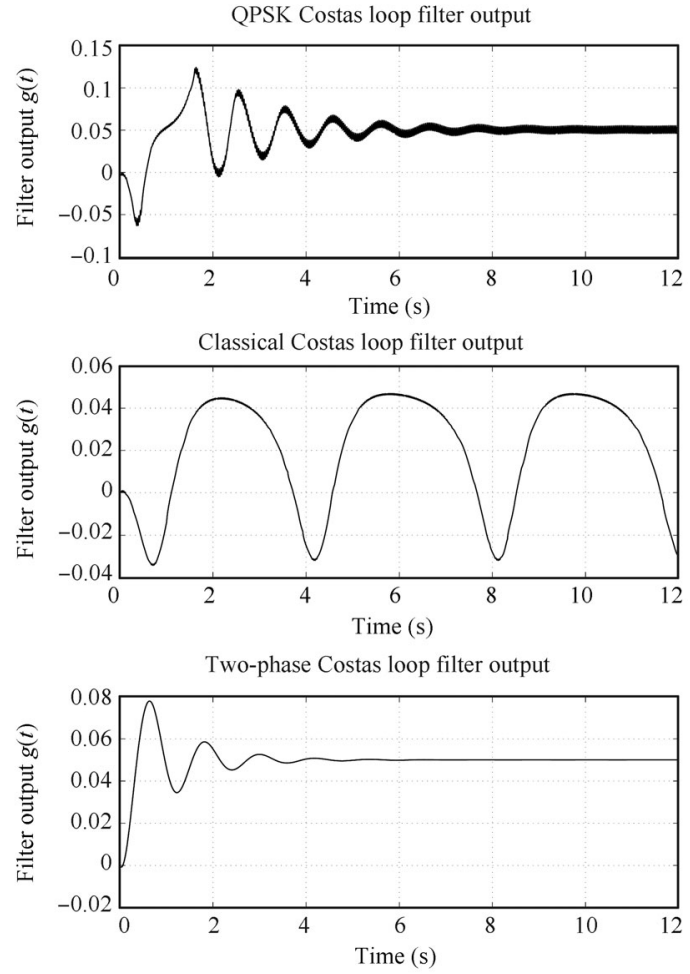

Fig. 9 Plot of the VCO input $g(t)$. VCO input gain: 30; lowpass filters transfer function: $\frac{1}{0.02 s+1}$; loop filter transfer function: $\frac{1}{s+2}$; VCO free-running frequency: 98.5 ; carrier frequency: 100
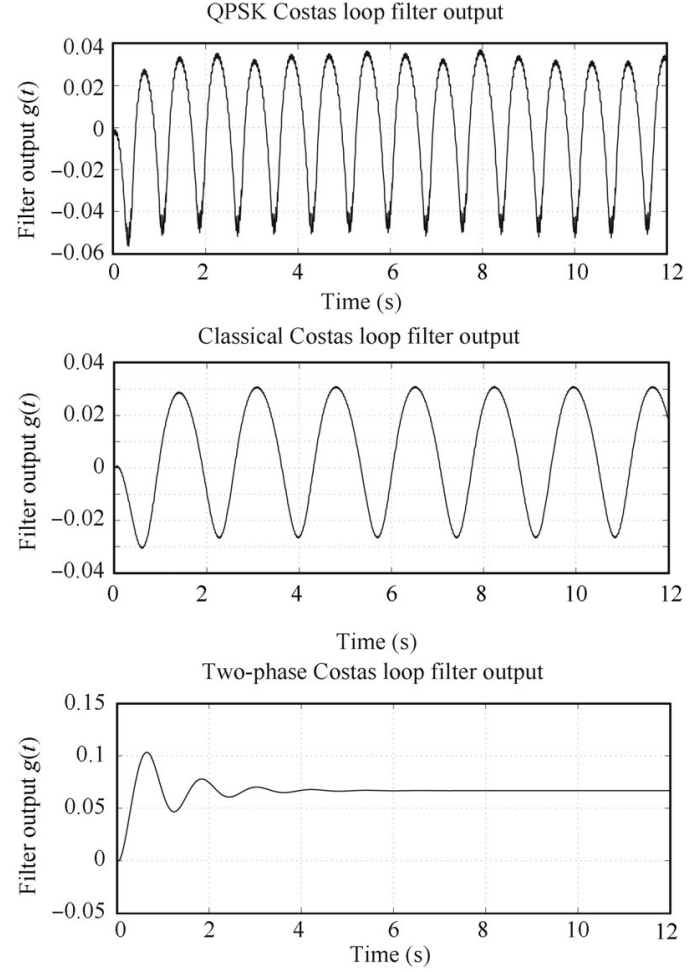

Fig. 10 Plot of the VCO input $g(t)$. VCO input gain: 30; lowpass filters transfer function: $\frac{1}{0.02 s+1}$; loop filter transfer function: $\frac{1}{s+2}$; VCO free-running frequency: 98; carrier frequency: 100
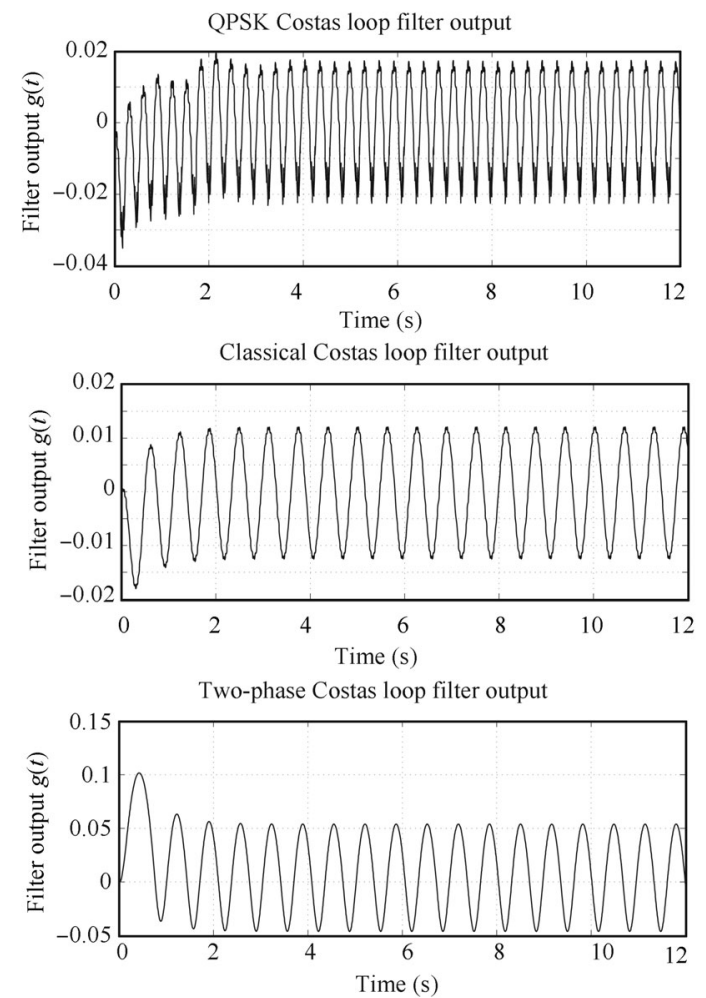

Fig. 11 Plot of the VCO input $g(t)$. VCO input gain: 30; lowpass filters transfer function: $\frac{1}{0.02 s+1}$; loop filter transfer function: $\frac{1}{s+2}$; VCO free-running frequency: 95 ; carrier frequency: 100

Note that unlike the loop filter input of the two-phase Costas loop, the input the filters of the classical and QPSK Costas loops contains additional high-frequency oscillations.

To analyze the high frequency signals accurately, a relatively large sampling frequency is required, which makes it difficult to perform a simulation in a reasonable time. In [51] one can read: "Direct time-domain simulation of PLLs at the level of SPICE circuits is typically impractical because of its great inefficiency. PLL transients can last hundreds of thousands of cycles, with each cycle requiring hundreds of small time steps for accurate simulation of the embedded voltage-controlled oscillator (VCO). Furthermore, extracting phase or frequency information, one of the chief metrics of PLL performance, from time-domain voltage/current waveforms is often difficult and inaccurate."

In the next section, we are going the discuss the mathematical approach that allows us to overcome this problem when analyzing Costas loops.

\section{Simulation of mathematical model of Costas loops in the signal's phase space}

The ideas behind are based on the works in $[3,52]$ and consist of the development of a mathematical model of PLLbased circuits in signal's phase space where only slow time scale of signal's phases is considered. Such an construction requires the computation of phase detector characteristic 
which depends on PD physical realization and the waveforms of the considered signals ${ }^{[53-60]}$.

Using the phase detector characteristic, instead of block diagrams in Figs. 1-3, one can consider the equivalent block diagram of Costas loop (Fig. 12) in the signal's phase space, where $\varphi(\theta)$ takes forms (1), (4), and (5), respectively.

In the equivalent block diagram, the loop filter has the same characteristic and initial state. The loop filter output signal $G(t)$ adjusts the VCO frequency to the frequency of input signal carrier.

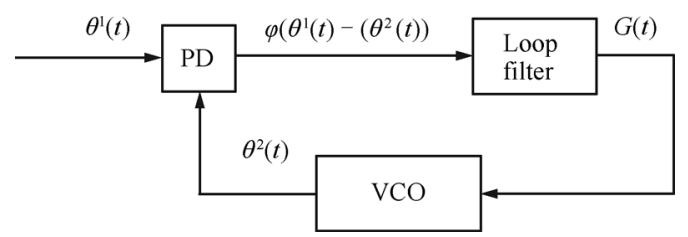

Fig. 12 Mathematical model of Costas loops in the signal's phase space

Note that construction of the mathematical model and using of analytic results for the conclusions on the behavior of the considered physical model need rigorous mathematical foundation ${ }^{[59,61]}$ : Here one has to prove that 1) $|g(t)-G(t)|$ is sufficiently small (without the assumption on ideality of the filters); 2) for the considered model of VCO frequency adjustment the behavior of the equivalent block diagrams are similar. The first requires the rigorous methods for phase detector characteristic computation ${ }^{[54,56,57,59,62-65]}$ and to prove the second it is essential to apply the averaging methods ${ }^{[66,67]}$.

\section{Mathematical foundation of math- ematical model for classical Costas loop}

The mathematical foundation of Costas loops consideration in the signal's phase space is considered.

The low-pass filters on the upper and the lower branches of the classical Costas loop (Fig. 1) are responsible for the demodulation process, therefore they can be applied separately from the loop (e.g., [9]. From a point of view of the analysis of stability, the filter at the input of VCO executes filtering functions. In this case, since $m(t)^{2}=1$, the transmitted data $m(t)$ does not affect the operation of the VCO. Thus one can consider the equivalent block diagrams of the classical Costas loop in signal/time and signal's phase spaces.

Consider a general case of the non-sinusoidal piecewisedifferentiable carrier oscillation $f^{1}\left(\theta^{1}(t)\right)$ and the tunable harmonic oscillation

$$
\begin{gathered}
f^{1}(\theta)=\sum_{i=1}^{\infty}\left(a_{i}^{1} \cos (i \theta)+b_{i}^{1} \sin (i \theta)\right) \\
f^{2}(\theta)=b_{1}^{2} \sin (\theta)
\end{gathered}
$$

where $f^{1}$ is represented as a Fourier series with coefficients $a^{1}$ and $b^{1}$.

Suppose that there exists a sufficiently large number $\omega_{\min }$ such that the following conditions are satisfied in a fixed time interval $[0, T]$ :

$$
\dot{\theta}^{p}(\tau) \geqslant \omega_{\min }>0, \quad p=1,2
$$

where $T$ is independent of $\omega_{\min }$, and $\dot{\theta}^{p}(\tau)=\frac{\mathrm{d} \theta^{p}(\tau)}{\mathrm{d} \tau}$ denotes the frequencies of signals. The frequencies difference is assumed to be uniformly bounded:

$$
\left|\dot{\theta}^{1}(\tau)-\dot{\theta}^{2}(\tau)\right| \leqslant \Delta \omega, \quad \forall \tau \in[0, T] .
$$

Requirements (7) and (8) are obviously satisfied for the tuning of two high-frequency oscillators with close frequencies. Denote $\delta=\omega_{\min }^{-\frac{1}{2}}$. Consider the following relations

$$
\begin{array}{r}
\left|\dot{\theta}^{p}(\tau)-\dot{\theta}^{p}(t)\right| \leqslant \Delta \Omega, \quad p=1,2 \\
|t-\tau| \leqslant \delta, \quad \forall \tau, t \in[0, T]
\end{array}
$$

where $\Delta \Omega$ is independent of $\delta$. Conditions (7)-(9) mean that the functions $\dot{\theta}^{p}(\tau)$ are almost constant and the functions $f^{p}\left(\theta^{p}(\tau)\right)$ are rapidly oscillating in small intervals $[t, t+\delta]$. Assume that

$$
|\gamma(\tau)-\gamma(t)|=\mathrm{O}(\delta), \quad|t-\tau| \leqslant \delta, \quad \forall \tau, t \in[0, T]
$$

where $\gamma(t)$ is an impulse response function of the loop filter.

The following assertion is valid.

Theorem $\mathbf{1}^{[65,68]}$. If conditions (7), (8), and (10) are satisfied and

$$
\begin{aligned}
\varphi(\theta)= & \frac{\left(b_{1}^{2}\right)^{2}}{8}\left[\left(a_{1}^{1}\right)^{2} \sin (2 \theta)+2 \sum_{q=1}^{\infty} a_{q}^{1} a_{q+2}^{1} \sin (2 \theta)-\right. \\
& 2 a_{1}^{1} b_{1}^{1} \cos (2 \theta)+2 \sum_{q=1}^{\infty} a_{q+2}^{1} b_{q}^{1} \cos (2 \theta)- \\
& 2 \sum_{q=1}^{\infty} a_{q}^{1} b_{q+2}^{1} \cos (2 \theta)-\left(b_{1}^{1}\right)^{2} \sin (2 \theta)+ \\
& \left.2 \sum_{q=1}^{\infty} b_{q}^{1} b_{q+2}^{1} \sin (2 \theta)\right]
\end{aligned}
$$

then the following relation is valid.

$$
G(t)-g(t)=\mathrm{O}(\delta), \quad \forall t \in[0, T] .
$$

In other words, this theorem separates the low-frequency error-correcting signal from parasitic high-frequency oscillations and proves that the considered function $\varphi(\theta)$ is a phase detector characteristic of Costas loop. For sinusoidal waveforms, without rigorous justification this fact was known to engineers ${ }^{[3]}$.

The details of the proof can be found in $[65,68]$. Note that this result could be easily extended to the case of two non-sinusoidal signals ${ }^{[69]}$. Arguing similarly, one can consider QPSK Costas loop for the sinusoidal signals ${ }^{[70]}$.

Well-known averaging method ${ }^{[66,67]}$ allows one to prove that the processes in both equivalent block diagrams of Costas loops in signal/time and signal's phase spaces are close under some assumption (see also simulations in Figs. 14 and 15).

To avoid using extremely high sampling rates, the physical model of Costas loop in the signal space can be replaced by the mathematical model in the signal's phase space that 
analyses only low frequency events (e.g. Simulink models in Figs. 5-7 can be replaced by the model in Fig. 13).

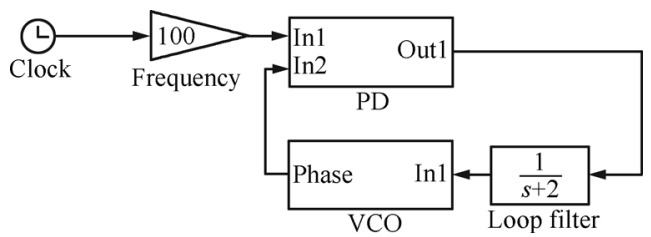

Fig. 13 Simulink model of Costas loops in signal's phase space

This allows one to overcome the difficulties mentioned above, and to study only slow time scale of signal's phases.
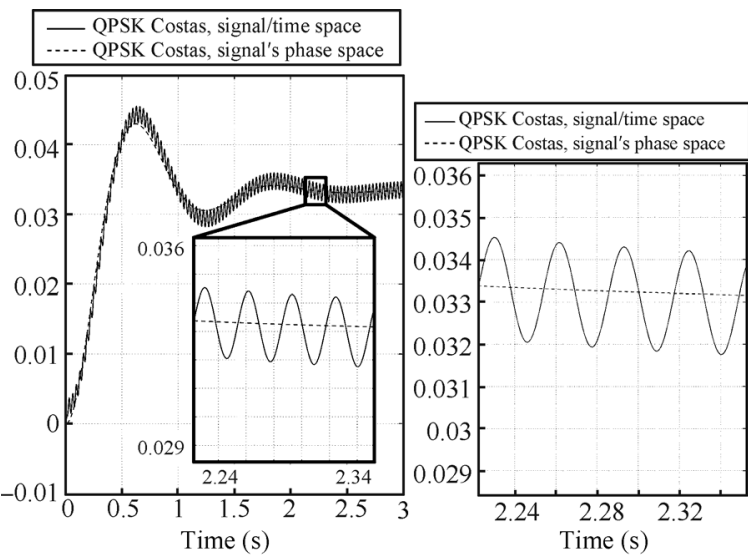

Fig. 14 Loop filter outputs $g(t)$ and $G(t)$. VCO input gain: 30; low-pass filters transfer function: $\frac{1}{0.02 s+1}$; loop filter transfer function: $\frac{1}{s+4}$; VCO free-running frequency: 99; carrier frequency 100

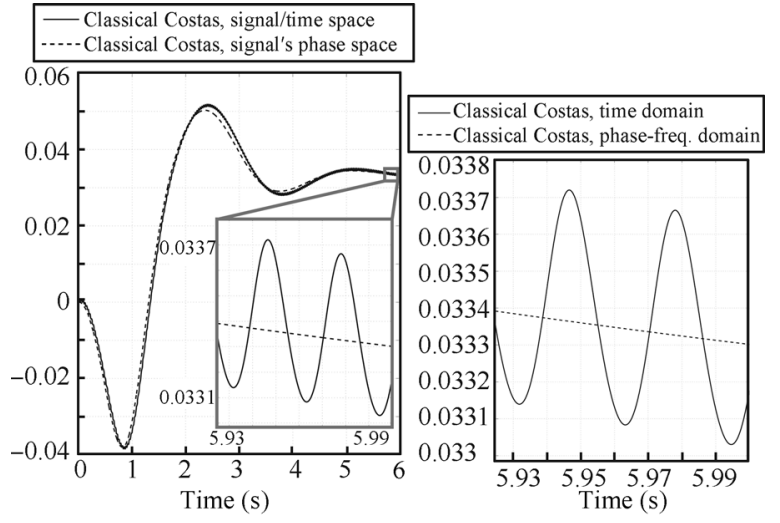

Fig. 15 Loop filter outputs $g(t)$ and $G(t)$. VCO input gain: 30; low-pass filters transfer function: $\frac{1}{0.02 s+1}$; loop filter transfer function: $\frac{1}{s+2}$; VCO free-running frequency: 99; carrier frequency: 100

\section{Conclusion}

To conclude, let us compare the time required to perform a simulation of the transient response of a classical Costas loop when either using the Simulink model for the signal space (see Figs. 5 and 6 ) or using the Simulink model for the phase space (see Fig. 13). Assume that the carrier frequency is $1 \mathrm{GHz}$. The duration of the transient response is shown to be about $0.4 \mathrm{~s}$. When the model for the signal space is used, the sampling frequency must be chosen (according to the Nyquist theorem) to be larger than twice the highest frequency existing in that system ${ }^{[16]}$.

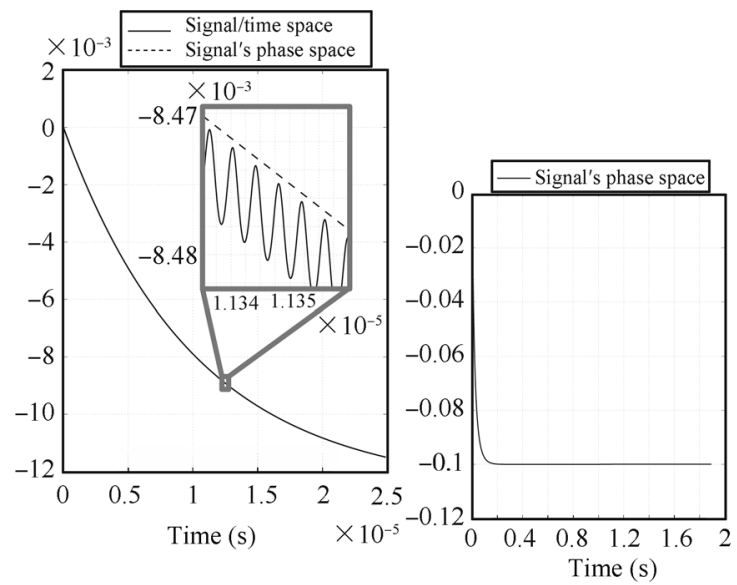

Fig. 16 Classical Costas loop filter output: VCO input gain: 200; loop filter transfer function: $\frac{1}{10^{-5} s+1}$; low-pass filters transfer functions: $\frac{1}{2 / 10^{9}+1}$; VCO free-running frequency: $10^{9}+20$; carrier frequency: $10^{9}$; discretization step for the signal space simulation: $10^{-10}$; discretization step for the signal's phase space simulation: $\frac{1}{200000}$; simulation time $=3 \mathrm{~s}$

As shown in Section 1.1.1, the maximum frequency is twice the carrier frequency, hence the sampling frequency must be chosen larger than $4 \mathrm{GHz}$. Using a sampling interval of $0.1 \mathrm{~ns}$, during $3 \mathrm{~s}$ of simulation only $25 \mu \mathrm{s}$ of the transient response was obtained.

When the model for the phase space is used, the sampling frequency can be chosen much lower. Using the phase space model, the whole transient could be simulated now in less than $0.4 \mathrm{~s}$, see Fig. 6 .

\section{References}

[1] R. E. Best. Phase Locked Loops: Design, Simulation, and Applications, 6th ed., New York: McGraw-Hill, 2007.

[2] Y. Shmaliy. Continuous-time Systems, Netherlands: Springer, 2007.

[3] F. M. Gardner. Phase-lock Techniques, New York: John Wiley and Son, 1966.

[4] W. C. Lindsey, M. K. Simón. Telecommunication Systems Engineering, New jersey: Prentice Hall, 1973.

[5] M. B. Pursley. Introduction to Digital Communications, New jersey: Prentice Hall, 2005.

[6] J. P. Costas. Synchronous communications. Proceedings of the IRE, vol. 44, no. 12, pp. 1713-1718, 1956.

[7] G. W. Waters. Costas Loop QPSK Demodulator, US Patent 4344178, USA, August 1982.

[8] I. B. Djordjevic, M. C. Stefanovic, S. S. Ilic, G. T. Djordjevic. An example of a hybrid system: Coherent optical system with Costas loop in receiver-system for transmission in 
baseband. Journal of Lightwave Technology, vol. 16, no. 2, pp. 177-183, 1998.

[9] E. D. Kaplan, C. J. Hegarty. Understanding GPS: Principles and Applications, Boston, USA: Artech House, 2006.

[10] D. Doberstein. Fundamentals of GPS Receivers: A Hardware Approach, New York: Springer, 2011.

[11] R. D. Stephens. Phase-Locked Loops for Wireless Communications: Digital, Analog and Optical Implementations, New York: Springer, 2002.

[12] Y. Wang, W. R. Leeb. A $90^{\circ}$ optical fiber hybrid for optimal signal power utilization. Applied Optics, vol. 26, no. 19, pp. 4181-4184, 1987 .

[13] T. Miyazaki, S. Ryu, Y. Namihira, H. Wakabayashi. Optical Costas loop experiment using a novel optical $90^{\circ}$ hybrid module and a semiconductor-laser-amplifier external phase adjuster. In Proceedings of Optical Fiber Communication, Optical Society of America, San Diego, California, pp. WH6, 1991.

[14] I. B. Djordjevic, M. C. Stefanovic. Performance of optical heterodyne PSK Systems with Costas loop in multichannel environment for nonlinear second-order PLL model. Journal of Lightwave Technology, vol. 17, no. 12, pp. 2470-2479, 1999.

[15] K. Hasegawa, H. Kanetsuna, M. Wakamori. GPS Positioning Method and GPS Reception Apparatus, EP Patent 1092987A2, USA, April 2001.

[16] P. S. Cho. Optical phase-locked loop performance in homodyne detection using pulsed and CW LO. In Proceedings of Optical Amplifiers and Their Applications/Coherent Optical Technologies and Applications, Optical Society of America, Whistler, Canada, pp. JWB24, 2006.

[17] Y. Hayami, F. Imai, K. Iwashita. Linewidth investigation for costas loop phase-diversity homodyne detection in digital coherent detection system. In Proceedings of the Asia Optical Fiber Communication and Optoelectronic Exposition and Conference, IEEE, Shanghai, China, pp. 1-3, 2008.

[18] G. M. Helaluddin. An improved optical costas loop PSK receiver: Simulation analysis. Journal of Scientific \& Industrial Research, vol. 67, pp. 203-208, 2008.

[19] N. Nowsheen, C. Benson, M. Frater. Design of a high frequency FPGA acoustic modem for underwater communication. In Proceedings of IEEE OCEANS, IEEE, Sydney, Australia, pp. 1-6, 2010.

[20] D. Abramovitch. Phase-locked loops: A control centric tutorial. In Proceedings of the 2002 American Control Conference, IEEE, Anchorage, AK, USA, vol. 1, pp. 1-15, 2002.

[21] D. Y. Abramovitch. Lyapunov Redesign of analog phaselock loops. IEEE Transactions on Communications, vol. 38, no. 12, pp. 2197-2202, 1990.

[22] K. Watada, T. Endo, H. Seishi. Shilnikov orbits in an autonomous third-order chaotic phase-locked loop. IEEE Transactions on Circuits and Systems I: Fundamental Theory and Applications, vol. 45, no. 9, pp.979-983, 1998.

[23] M. Hinz, I. Konenkamp, E. H. Horneber. Behavioral modeling and simulation of phase-locked loops for RF front ends. In Proceedings of the 43rd IEEE Midwest Symposium on Circuits and Systems, IEEE, Lansing, MI, USA, pp. 194$197,2000$.
[24] A. Rantzer. Almost global stability of phase-locked loops. In Proceedings of the 40th IEEE Conference on Decision and Control, IEEE, Orlando, FL, USA, vol. 1, pp. 899-900, 2001.

[25] N. E. Wu. Analog phaselock loop design using Popov criterion. In Proceedings of the 2002 American Control Conference, IEEE, Anchorage, AK, USA, vol. 1, pp. 16-18, 2002.

[26] J. R. C. Piqueira, L. H. A. Monteiro. Considering secondharmonic terms in the operation of the phase detector for second-order phase-locked loop. IEEE Transactions on Circuits and Systems I: Fundamental Theory and Applications, vol. 50, no. 6, pp. 805-809, 2003.

[27] A. Suarez, R. Quere. Stability Analysis of Nonlinear Microwave Circuits, New Jersey: Artech House, 2003.

[28] W. I. Margaris. Theory of the Non-Linear Analog Phase Locked Loop, New Jersey: Springer Verlag, 2004.

[29] G. D. Vendelin, A. M. Pavio, U. L. Rohde. Microwave Circuit Design Using Linear and Nonlinear Techniques, New York: Wiley, 2005.

[30] P. Goyal, X. L. Lai, J. Roychowdhury. A fast methodology for first-time-correct design of PLLs using nonlinear phasedomain VCO macromodels. In Proceedings of the 2006 Asia and South Pacific Conference on Design Automation, IEEE, Yokohama, Japan, pp. 291-296, 2006.

[31] V. V. Matrosov. Nonlinear dynamics of phase-locked loop with the second-order filter. Radiophysics and Quantum Electronics, vol. 49, no. 3, pp. 239-249, 2006.

[32] J. Kudrewicz, S. Wasowicz. Equations of Phase-locked Loops: Dynamics on the Circle, Torus and Cylinder, vol. 59, Singapore: World Scientific Publishing Company, 2007.

[33] O. Feely. Nonlinear dynamics of discrete-time circuits: A survey. International Journal of Circuit Theory and Applications, no. 35 , no. 5-6, pp. 515-531, 2007.

[34] N. V. Kuznetsov. Stability and Oscillations of Dynamical Systems: Theory and Applications, Jyväskylä: Jyväskylä University Printing House, 2008.

[35] T. C. Wang, T. Y. Chiou, S. Lall. Nonlinear Phase-locked Loop design using semidefinite programming. In Proceedings of the 16th Mediterranean Conference on Control and Automation, IEEE, Ajaccio, France, pp. 1640-1645, 2008.

[36] J. Stensby. VCO sweep-rate limit for a phase-lock loop. Journal of the Franklin Institute, vol. 346, no. 3, pp. 223236, 2009.

[37] C. Wiegand, C. Hedayat, U. Hilleringmann. Non-linear behaviour of charge-pump phase-locked loops. Advances in Radio Science, vol. 8, pp. 161-166, 2010.

[38] P. F. Curran, C. Bi, O. Feely. Dynamics of charge-pump phase-locked loops. International Journal of Circuits Theory and Applications, vol.41, no. 11, pp. 1109-1135, 2013.

[39] A. Suarez, E. Fernandez, F. Ramirez, S. Sancho. Stability and bifurcation analysis of self-oscillating quasi-periodic regimes. IEEE Transactions on Microwave Theory and Techniques, vol. 60, no. 3, pp. 528-541, 2012.

[40] T. Banerjee, B. C. Sarkar. Conventional and extended time-delayed feedback controlled zero-crossing digital phase locked loop. International Journal of Bifurcation and Chaos, vol. 22 , no. 12 , pp. $1230044,2012$. 
[41] A. S. Huque, J. Stensby. An analytical approximation for the pull-out frequency of a PLL employing a sinusoidal phase detector. ETRI Journal, vol. 35, no. 2, pp. 218-225, 2013.

[42] C. Chicone, M. T. Heitzman. Phase-locked loops, demodulation, and averaging approximation time-scale extensions. SIAM Journal on Applied Dynamical Systems, vol. 12, no. 2, pp. 674-721, 2013.

[43] M. C. Jeruchim, P. Balaban, K. S. Shanmugan. Simulation of Communication Systems: Modeling, Methodology and Techniques, New York: Springer, 2000.

[44] D. Banerjee. PLL Performance, Simulation and Design, 4th ed., USA: Dog Ear Publishing, 2006.

[45] D. O. Pederson, K. Mayaram. Analog Integrated Circuits for Communication: Principles, Simulation and Design, 2nd edition, New York: Springer, 2008.

[46] W. Tranter, T. Bose, R. Thamvichai. Basic Simulation Models of Phase Tracking Devices Using MATLAB. Synthesis Lectures on Communications, San Rafael, CA, USA: Morgan \& Claypool Publishers, 2010.

[47] A. Al-Harasees, G. O. Al-Maaitah. Overshoot Improvement for Indirect Frequency Synthesizer Phase Locked Loop. International Journal of Electrical, Electronics and Computer Systems, vol. 15, no. 1, pp. 1011-1023, 2013.

[48] D. Y. Abramovitch. Efficient and flexible simulation of phase locked loops, Part I: Simulator design. In Proceedings of the 2008 American Control Conference, IEEE, Seattle, WA, USA, pp. 4672-4677, 2008.

[49] D. Y. Abramovitch. Efficient and flexible simulation of phase locked loops, Part II: Post processing and a design example. In Proceedings of the 2008 American Control Conference, IEEE, Seattle, WA, USA, pp. 4678-4683, 2008.

[50] S. A. Tretter. Communication System Design Using DSP Algorithms: With Laboratory Experiments for the TMS320C6713TM DSK, New York: Springer, 2008.

[51] X. L. Lai, Y. Y. Wan, J. S. Roychowdhury. Fast PLL simulation using nonlinear VCO macromodels for accurate prediction of jitter and cycle-slipping due to loop non-idealities and supply noise. In Proceedings of the 2005 Asia and South Pacific Design Automation Conference, ACM Press, New York, USA, pp. 459-464, 2005.

[52] A. Viterbi. Principles of Coherent Communication, New York: McGraw-Hill, 1966.

[53] G. A. Leonov. Computation of phase detector characteristics in phase locked loops for clock synchronization. Doklady Mathematics, vol. 78, no. 1, pp. 643-645, 2008.

[54] N. V. Kuznetsov, G. A. Leonov, S. M. Seledzhi. Nonlinear analysis of the Costas loop and phase-locked loop with squarer. In Proceedings of the IASTED International Conference on Signal and Image Processing, Honolulu, Hawaii, USA, pp. 1-7, 2009.

[55] N. V. Kuznetsov, G. A. Leonov, P. Neittaanmäki, S. M. Seledzhi, M. V. Yuldashev, R. V. Yuldashev. Nonlinear analysis of phase-locked loop. In Proceedings of the 4th IFAC International Workshop on Periodic Control Systems, IFAC, Turkey, pp. 34-38, 2010.
[56] N. V. Kuznetsov, G. A. Leonov, P. Neittaanmäki, S. M. Seledzhi, M. V. Yuldashev, R. V. Yuldashev. Highfrequency analysis of phase-locked loop and phase detector characteristic computation. In Proceedings of the 8th International Conference on Informatics in Control, Automation and Robotics, SciTePress, the Netherlands, vol. 1, pp. 272$278,2011$.

[57] N. V. Kuznetsov, G. A. Leonov, M. V. Yuldashev, R. V. Yuldashev. Analytical methods for computation of phasedetector characteristics and PLL design. In Proceedings of the 10th International Symposium on Signals, Circuits and Systems, IEEE, Iasi, Romania, pp. 1-4, 2011.

[58] N. V. Kuznetsov, G. A. Leonov, P. Neittaanmäki, S. M. Seledzhi, M. V. Yuldashev, R. V. Yuldashev. Nonlinear mathematical models of Costas Loop for general waveform of input signal. In Proceedings of the 4th IEEE International Conference on Nonlinear Science and Complexity, IEEE, Budapest, Hungary, pp. 75-80, 2012.

[59] G. A. Leonov, N. V. Kuznetsov, M. V. Yuldahsev, R. V. Yuldashev. Analytical method for computation of phasedetector characteristic. IEEE Transactions on Circuits and Systems II: Express Briefs, vol.59, no. 10, pp.633-637, 2012.

[60] R. E. Best, N. V. Kuznetsov, G. A. Leonov, M. V. Yuldashev, R. V. Yuldashev. Discontinuity and complexity in nonlinear physical systems. Nonlinear Systems and Complexity, vol. 6, New York: Springer, 2014.

[61] G. A. Leonov, N. V. Kuznetsov, M. V. Yuldahsev, R. V. Yuldashev. Computation of phase detector characteristics in synchronization systems. Doklady Mathematics, vol. 84, no. 1 , pp. 586-590, 2011.

[62] G. A. Leonov, N. V. Kuznetsov, S. M. Seledzhi. Analysis of phase-locked systems with discontinuous characteristics. In Proceedings of the 1st IFAC Conference on Analysis and Control of Chaotic Systems, IFAC, France, vol. 1, pp. 107$112,2006$.

[63] N. V. Kuznetsov, G. A. Leonov, S. S. Seledzhi. Phase locked loops design and analysis. In Proceedings of the 5th International Conference on Informatics in Control, Automation and Robotics, pp. 114-118, 2008.

[64] N. V. Kuznetsov, G. A. Leonov, P. Neittaanmäki, S. M. Seledzhi, M. V. Yuldashev, R. V. Yuldashev. Simulation of phase-locked loops in phase-frequency domain. In Proceedings of the 4th International Congress on Ultra Modern Telecommunications and Control Systems and Workshops, IEEE, St. Petersburg, Russia, pp. 351-356, 2012.

[65] N. V. Kuznetsov, G. A. Leonov, M. V. Yuldashev, R. V. Yuldashev. Nonlinear analysis of Costas loop circuit. In Proceedings of the 9th International Conference on Informatics in Control, Automation and Robotics, IFAC, Rome, Italy, vol. 1, pp. 557-560, 2012.

[66] N. Krylov, N. Bogolyubov. Introduction to Non-linear Mechanics, Princeton: Princeton University Press, 1947.

[67] Y. A. Mitropolsky, N. Bogolubov. Asymptotic Methods in the Theory of Non-linear Oscillations, New York: Gordon and Breach, 1961.

[68] G. A. Leonov, N. V. Kuznetsov, M. V. Yuldashev, R. V. Yuldashev. Differential equations of Costas loop. Doklady Mathematics, vol. 86, no. 2, pp. 723-728, 2012. 
[69] N. V. Kuznetsov, G. A. Leonov, M. V. Yuldashev, R. V. Yuldashev. Analytical computation of phase-detector characteristics and dynamical model of Costas loop for nonsinusoidal signals. IEEE Transactions on Circuits and Systems $I$, to be published.

[70] G. A. Leonov, N. V. Kuznetsov, M. V. Yuldashev, R. V. Yuldashev. Analytical computation of phase-detector characteristics and dynamical model of QPSK Costas loop. Doklady Mathematics, to be published.

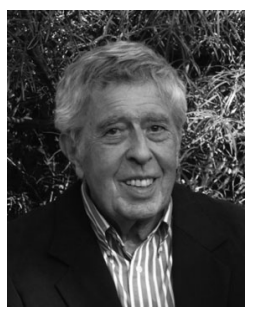

Roland E. Best received his Ph. D. degree in electrical engineering from Swiss Federal Institute in Zurich (ETHZ). He worked 3 years at IBM research laboratory in Zurich, Switzerland. Then he entered to the Sandoz company (now Novartis) as a project engineer and carried out numerous automation projects for dystuffs, pharmaceutical and agrochemical production plants. After retirement he founded his private company Best Engineering which is mainly active in project engineering, consulting and organizing seminars and courses.

He is the author of many scientific publications and a number of books. The book "Roland E. Best, Phase-locked Loops, Design, Simulation, and Applications, McGraw-Hill, New York" was published first in 1984, and the 6th edition appeared in 2007. This book is considered a standard textbook in this field. Because it was also very successful in south-eastern Asia, a Chinese translation has been provided.

His research interests include design, simulation, and applications of phase-locked loops and Costasloops.

E-mail: Rolandbest@aol.com

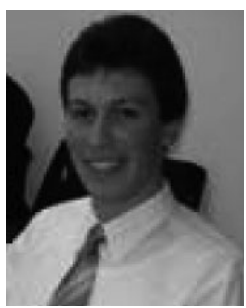

Nikolay V. Kuznetsov received his candidate of science degree from SaintPetersburg State University, Russia in 2004 and Ph. D. degree from University of Jyväskylä, Finland in 2008. He is currently deputy head of Applied Cybernetics Department at Saint-Petersburg University, Russia, and coordinator of FinnishRussian Educational \& Research Program, organized by University of Jyväskylä and Saint-Petersburg University. He is the author and co-author of more than 100 publications.
His research interests include stability by the first approximation and Lyapunou exponent sign reversal, hidden attractors and chaos, describing function method and absolute stability, nonlinear analysis and design of PLL-based circuits.

E-mail: nkuznetsov239@gmail.com (Corresponding author)

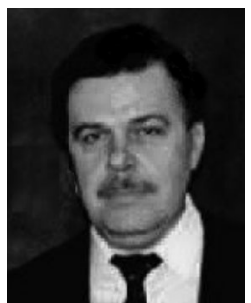

Gennady A. Leonov received his Ph. D. degree in 1971 and Doctor of Science in 1983 from Saint-Petersburg State University, Russia. Now, he is dean of Mathematics and Mechanics Faculty (since 1988), director of Research Institute of Mathematics and Mechanics of Saint-Petersburg State University (since 2004), head of Applied Cybernetics Department (since 2007). He was awarded Prize of Saint-Petersburg State University (1985), State Prize of USSR (1986), Prize of Technische Universitet Dresden (1990), Medal of University of Jyväskylä (2011), Andronov prize of Russian Academy of Science (2012). He is a member (corresponding) of Russian Academy of Science, Russian National Committees of Theoretical Mechanics and Automatic control, Directorate of Saint-Petersburg Mathematical Society. In 2011, he was elected to International Federation of Automatic Control (IFAC) Council.

His research interests, include control theory, dynamical systems and phase synchronization systems.

E-mail: leonov@math.spbu.ru

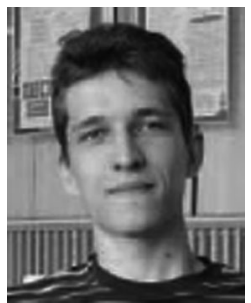

Marat V. Yuldashev received his Ph. D. degree from Saint-Petersburg State University, Russia in 2013. He is currently an associate professor in Saint-Petersburg University.

His research interests include nonlinear models of Costas loops.

E-mail:maratyv@gmail.com

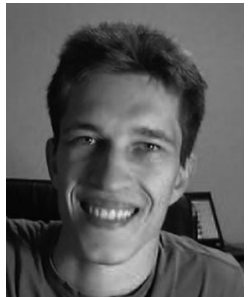

Renat V. Yuldashev received his Candidate degree from Saint-Petersburg State University, Russia in 2013. He is currently an associate professor in Saint-Petersburg University.

His research interests include nonlinear models of phase-locked loops.

E-mail: renatyv@gmail.com 